\title{
Complete Removal of the Infrapatellar Fat Pad in Total Knee Arthroplasty Does Not Affect the Patellar Height
}

\author{
Serkan Güler' (1), Sercan Capkin' (1), Ramadan Özmanevra² (1) \\ 'Department of Orthopedics and Traumatology, Aksaray University School of Medicine, Aksaray, Turkey \\ 2Department of Orthopedics and Traumatology, University of Kyrenia, School of Medicine, Kyrenia, Cyprus
}

ORCID iDs of the authors: S.G. 0000-0002-3424-4244; S.C. 0000-000I-6957-5927; R.0̈. 0000-0003-05I5-400I.

Cite this article as: Güler S, Capkin S, Özmanevra R. Complete Removal of the Infrapatellar Fat Pad in Total Knee Arthroplasty Does Not Affect the Patellar Height. Cyprus J Med Sci 2020; 5(4): 350-4.

\section{BACKGROUND/AIMS}

Most orthopedic surgeons tend to remove the infrapatellar fat pad (IPFP) in total knee arthroplasty (TKA). The present study was designed with the aim of determining whether complete removal of the IPFP during TKA affects the patella height.

\section{MATERIAL and METHODS}

Knee radiographs of 85 patients who underwent primary TKA that involved complete IPFP removal were reviewed retrospectively. We measured the patella height using the Insall-Salvati (IS) ratio, modified IS (mIS) ratio, Cathon-Deschamp (CD) index, and BlackburnePeel (BP) index on preoperative radiographs and at the l-year follow-up. Two orthopedic surgeons independently analyzed all 85 radiographs and were blinded to the measurement taken by the other surgeon. The average values of the data recorded by the two observers was used in the final analyses.

\section{RESULTS}

The average preoperative patella height was higher than the mean patella height at I year after TKA using all the measurement methods. Although this may reflect patellar tendon shortening or elevation of the joint line, this decrease was not significant using all methods $(p>0.05)$.

\section{CONCLUSION}

Complete removal of the IPFP during TKA for patients with primary osteoarthritis did not affect the patellar height at the I-year follow-up.

Keywords: Total knee arthroplasty, fat pad, height, patella, complete, removal

\section{INTRODUCTION}

Hoffa's fat pad, commonly called the infrapatellar fat pad (IPFP), is an extra-synovial structure that consists of fibrous adipose tissue and is located between the synovium and joint capsule (I). Generally, it is partially or completely removed during total knee arthroplasty (TKA) to allow better visualization of the lateral compartment of the joint $(2,3)$. However, the results of IPFP removal are unclear, and the issue of preserving or removing the IPFP remains controversial (4). IPFP removal may cause shortening of the patellar tendon, increase anterior knee pain, and reduce flexion (2-5). Complete removal of IPFP during TKA may cause injury to the lateral genicular artery and damage the vascular supply of the patellar tendon, causing tendon scarring (5). Patellar tendon shortening and scarring of the IPFP can result in a patella baja after TKA $(3,5)$. Patella baja (PB) is identified by abnormally low placement of the patella on lateral radiography compared to the femoral trochlea. Reduced patella height is a common finding after TKA and is mainly caused by shortening of the patellar tendon or elevation of the joint line (6). In the literature, various methods have been suggested to measure the height of the patella using pre-TKA and post-TKA radiography (7).

Patellar tendon shortening is a known complication after TKA; however, there is no consensus about the role perioperative IPFP removal in patellar tendon shortening in the literature (8). Some previous studies have reported that the patellar tendon shortened after IPFP removal, while others have shown no effect of IPFP removal on the patellar tendon length 
$(2,5,9-11)$. Considering the increase in the number of patients undergoing TKA, there is no consensus on the effect of complete removal of the IPFP on patellar tendon shortening that reflects as a decrease in the patellar height. Therefore, we designed this study to determine whether complete removal of the IPFP during TKA affects the patella height before and after TKA. We hypothesized that complete removal of the IPFP may affect the patella height after TKA.

\section{MATERIAL and METHODS}

We retrospectively reviewed the electronic medical records of II2 patients who underwent primary TKA from January 2017 to April 2019 for potential inclusion in the study. We enrolled patients of any age and sex who had undergone primary TKA for primary knee osteoarthritis and for whom non-weight-bearing lateral knee radiographs taken in at at least $30^{\circ}$ flexion preoperatively and first-year postoperatively were available. Patients with lateral release, patellar resurfacing, any other prior surgery in the same knee, ; any systemic inflammatory disease, ; a history of revision surgery for periprosthetic infection, fracture, or other causes, ; bilateral TKA, ; and patellar tendon injury during TKA who had undergone TKA for causes other than primary osteoarthritis were excluded. We also excluded patients for whom knee radiographs performed at at least $30^{\circ}$ flexion before and after the operation were available and those whose knees were over-rotated, whose radiographs were not visible, and those who disrupted the correct measurement of patellar height. Furthermore, we did not include the data of patients who died during the postoperative follow-up period or were lost to follow-up. Of the II2 patients who were identified as potential study subjects, finally, 27 were excluded as per these criteria. Thus, the data of 85 patients were included in the final analyses Ethical approval was obtained from the local ethics committee Aksaray University Human Research Ethical Committee (2020/06-12), and the need for informed consent of the patients was waived owing to the retrospective nature of the study.

\section{Surgical Technique}

All the surgical procedures were performed by the first author under spinal anesthesia (S.G.). In all the procedures, a medial parapatellar approach was preferred, a tourniquet was used, and the IPFP was removed completely for all patients. The prosthesis used in all the patients was the Vanguard ${ }^{\circledR}$ Complete

\section{Main Points:}

- Hoffa's fat pad, commonly known as the infrapatellar fat pad (IPFP) is an extra-synovial structure that consists of fibrous adipose tissue and is partially or completely removed during total knee arthroplasty (TKA) to allow better visualization of the lateral compartment of the joint.

- The results of IPFP removal are unclear, and the issue of preserving or removing the IPFP remains controversial.

- Patellar tendon shortening is a known complication after TKA; however, there is no consensus about the contribution of IPFP removal during surgery in the literature.

- We conclude that complete removal of the IPFP during TKA for primary osteoarthritis did not affect the patellar height at the l-year follow-up.
Knee System manufactured by Zimmer Biomet in Warsaw, Indiana. A cemented TKA that has fixed-bearing insert and sacrificing posterior cruciate ligament were used in all patients. The patellar surface was not replaced in any patient.

\section{Assessment of Radiologic Parameters}

Radiological measurements were performed using the lateral view of the knee at $30^{\circ}$ flexion preoperatively and at I y postoperatively. All the measurements were obtained electronically using lateral knee radiographs with the picture archiving and communication system (Infinity Healthcare Co., Seoul, South Korea). Two orthopedic surgeons independently analyzed all 85 radiographs and were blinded to each other's measurements. The average values of the data recorded by the two surgeons were used for the final analyses. The patella height was measured on radiographs using the Insall-Salvati (IS) ratio, modified IS ( $\mathrm{mlS}$ ) ratio, Blackburne-Peel (BP) index, and CatonDeschamps (CD) index. In order to calibrate the patellar height measurements and minimize enlargement errors, the diameter of the femoral shaft was measured as a constant value at $8 \mathrm{~cm}$ proximal to the combination of the condyles and femoral shaft on each lateral radiograph, as previously described (2).

For each radiograph, the preoperative patellar height was measured using the following four distinct methods: IS, mIS, BP, and $C D$, as described in their respective original publications (12-15). The IS includes the ratio of the length of the patellar tendon (the distance between the distal pole of the patella and the tibial tuberosity) to the maximum length of the patella (between the distal pole and the proximal pole of the patella) (Figure la). Normal values range from 0.8 to 1.2 (I2). The mIS ratio consists of the ratio of the distance between the tibial tuberosity and the articular surface of the patellar distal pole to the length of the articular surface of the patella (Figure lb). A ratio $>2$ indicates a patella alta (13). The BP index is the ratio of the perpendicular height between the anterior superior corner of the tibia and the inferior aspect of the patellar articular surface to the length of the articular surface of the patella (Figure Ic). Normal values range from 0.54 to 1.06 (14). The CD index comprises the ratio of the distance between the antero-superior point of the tibial plateau and the distal pole of the patellar articular surface to the length of the articular surface of the patella (Figure Id). Normal values range from 0.6 to 1.2 (I5).

IS and mIS ratios were measured at I year postoperatively using the same method that was used preoperatively (Figure le, f). Postoperative CD and BP indices were measured using the modified versions because they were more applicable for measuring the patella height after TKA $(7,16)$. The modified BP (mBP) index was defined as the vertical height divided by the antero-superior corner of the polyethylene insert to the lower direction of the patellar joint surface and the length of the joint surface of the patella (Figure $\mathrm{lg}$ ). Normal values range from 0.54 to 1.06 (16). The modified CD ( $\mathrm{mCD}$ ) index is calculated by dividing the patella by the length of the joint surface between the lower end of the joint surface and the antero-superior corner of the tibial polyethylene insert (Figure Ih). Normal values range from 0.6 to 1.2 (7). These modifications enable the measurement of the height of the patella from the joint line; therefore, they are less affected by the thickness of the proximal tibial resection and tibial polyethylene insert $(7,16)$. 


\section{Statistical Analysis}

Statistical analyses were performed using the Statistical Package for Social Sciences software version 20.0 (IBM SPSS Corp.; Armonk, NY, USA). Descriptive statistics are presented as mean, standard deviation, and range values, as well as percentages. The inter-observer reliability for the two observers was determined with intra-class correlation coefficients (ICC). The scores were interpreted as follows: a score of 0-0.50 indicated poor reliability, 0.50-0.75 indicated moderate reliability, 0.75-0.90 indicated good reliability, and a score $>0.90$ showed excellent reliability (17). The data were tested for normality using the Shapiro-Wilks test. Paired t test was used to assess the statistical significance of the differences between the preoperative and postoperative measurements. A $p$ - value of $<0.05$ was considered significant. The most important results are presented in the tables.

\section{RESULTS}

Among the 85 patients included in this study, 17 were men and 68 were women. At the time of the surgery, the average age of the study subjects was $66.96 \pm 8.42$ y (range, $50-83$ y), and their average body mass index was $27.47 \pm 1.59 \mathrm{~kg} / \mathrm{m}^{2}$ (range, 24.68$\left.32.12 \mathrm{~kg} / \mathrm{m}^{2}\right)$.

With regard to the preoperative measurements, moderate inter-observer reliability had an ICC of 0.74 ( $95 \%$ confidence interval [Cl], 0.58-0.82) for BP index, while other measurements

TABLE I. Interobserver reliability for the measurements of patellar height during the preoperative period

\begin{tabular}{|lccc|} 
Methods & ICC & $95 \% \mathrm{Cl}$ & p value \\
\hline Insall-Salvati & 0.88 & $0.84-0.93$ & $<0.001$ \\
Modified Insall-Salvati & 0.77 & $0.63-0.85$ & $<0.001$ \\
Caton-Deschamps & 0.81 & $0.72-0.87$ & $<0.001$ \\
Blackburne-Peel & 0.74 & $0.58-0.82$ & $<0.001$ \\
\hline ICC: intra-class correlation coefficients, Cl: confidence interval & \\
\hline
\end{tabular}

TABLE 2. Interobserver reliability for the measurements of patellar height at postoperative

\begin{tabular}{|lccc|}
\hline Methods & ICC & $95 \% \mathrm{Cl}$ & p value \\
\hline Insall-Salvati & 0.89 & $0.83-0.93$ & $<0.001$ \\
Modified Insall-Salvati & 0.76 & $0.63-0.84$ & $<0.00$ I \\
Modified Caton-Deschamps & 0.84 & $0.76-0.89$ & $<0.00$ I \\
Modified Blackburne-Peel & 0.70 & $0.54-0.80$ & $<0.00$ I \\
\hline ICC: intra-class correlation coefficients, Cl: confidence interval & \\
\hline
\end{tabular}

TABLE 3. Comparison of mean patellar heights before and after TKA

\begin{tabular}{|c|c|c|c|}
\hline Methods & Preoperative & Postoperative & $p$ value \\
\hline IS & $1 \pm 0.12(0.75-1.33)$ & $0,98 \pm 0.09(0.79-1.2)$ & 0.203 \\
\hline $\mathrm{mIS}$ & $1.57 \pm 0.17(1.26-1.99)$ & $1.55 \pm 0.17(1.17-2)$ & 0.45 \\
\hline $\mathrm{CD} / \mathrm{mCD}$ & $0.82 \pm 0.13(0.49-1.14)$ & $0.81 \pm 0.12(0.51-1.06)$ & 0.809 \\
\hline $\mathrm{BP} / \mathrm{mBP}$ & $0.77 \pm 0.12(0.47-1.03)$ & $0.74 \pm 0.1(0.48-0.95)$ & 0.081 \\
\hline
\end{tabular}

(IS, mIS, and CD) had good inter-observer reliability (Table I). With regard to the postoperative measurements, moderate inter-observer reliability was shown with an ICC of $0.70(95 \% \mathrm{Cl}$, 0.54-0.80) for the mBP index; the other measurements (IS, mIS, and $\mathrm{mCD}$ ) had good inter-observer reliability (Table 2).

Table 3 presents a comparison of the mean patellar heights before and after TKA. Shapiro-Wilks test was used to determine whether the data were normally distributed. Therefore, paired $t$ test was used to assess the statistical significance of the differences between the preoperative and postoperative measurements.

The mean IS ratio preoperatively was $1 \pm 0.12$, and this ratio decreased to $0.98 \pm 0.09$ at the l-year follow-up. The reduction in the average IS ratio was not significant $(p=0.203)$. The IS ratio remained unchanged in $4(4.70 \%)$ patients, decreased in 54 $(63.53 \%)$, and increased in 27 (31.76\%). The average preoperative $\mathrm{mIS}$ ratio was $1.57 \pm 0.17$, and this ratio decreased to $1.55 \pm 0.17$ at the l-year follow-up. The reduction in the mean $\mathrm{mIS}$ ratio was not significant $(p=0.45)$. The $\mathrm{mIS}$ ratio remained unchanged in $4(4.71 \%)$ patients, decreased in $52(61.18 \%)$, and increased in 29 (34.11\%). The average preoperative CD index was $0.82 \pm 0.13$, and this value decreased to $0.81 \pm 0.12(m C D)$ at the l-year follow-up. The reduction in the mean CD index was not significant $(p=0.809)$. The CD index remained unchanged in II (I2.94\%) patients, decreased in 42 (49.41\%), and increased in 32 (37.6\%).

The average preoperative $\mathrm{BP}$ index was $0.77 \pm 0.12$, and this value decreased to $0.74 \pm 0.1$ (mBP) at the l-year follow-up. The reduction in the mean BP index was not significant $(p=0.081)$. The BP

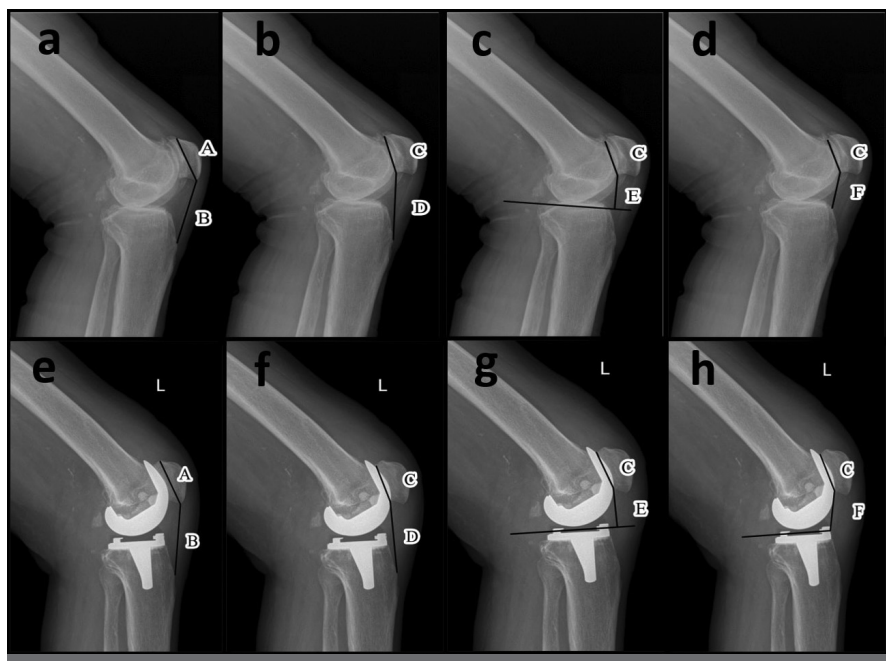

Figure I. a-h. Representation of the four methods used for measuring patellar height. (a) Preoperative Insall-Salvati ratio: B/A, (b) Preoperative Modified Insall-Salvati ratio: D/C, (c) Preoperative Blackburne-Peel index: E/C, (d) Preoperative Caton-Deschamps index: F/C, (e) Postoperative Insall-Salvati ratio: B/A, f. Postoperative Modified Insall-Salvati ratio: D/C, (g) Postoperative Modified Blackburne-Peel index: E/C, (h) Postoperative Modified Caton-Deschamps index: $\mathrm{F} / \mathrm{C}$

A: The diagonal length of the patella, B: The length of the patellar tendon $C$ : The length of the articular surface of the patella, $D$ : The distance between the lower end of the articular surface of the patella and patellar tendon insertion to the tibial tubercle, E: The perpendicular height from the anterior superior corner of the tibial polyethylene insert to the inferior aspect of the patellar articular surface, $F$ : The distance between the lower end of the articular surface of the patella and the anterior superior corner of the tibial polyethylene insert. 
index remained unchanged in $5(5.88 \%)$ patients, decreased in $46(54.12 \%)$, and increased in $34(40 \%)$.

According to the preoperative IS ratio, $82(96.4 \%)$ subjects had a normal patellar height, I (I.2\%) had a PB, and $2(2.4 \%)$ had a patella alta. As per the preoperative mIS ratio, $\mathrm{PB}$, or alta was not detected in 85 patients. As per the preoperative CD index, 83 (97.6\%) patients had a normal patellar height, while 2 $2.4 \%$ ) had a PB. Based on the preoperative BP index, 8 I (95.2\%) patients had a normal patellar height, $2(2.4 \%)$ had a PB, and $2(2.4 \%)$ had a patella alta.

According to the postoperative IS ratio, 84 (98.8\%) patients had normal patellar height, while I (I.2\%) subjects had a PB. According to the postoperative mIS ratio, 84 (98.8\%) had a normal patellar height, while I (I.2\%) had a PB. As per the postoperative $\mathrm{mCD}$ index, 83 (97.6\%) subjects had normal patellar height, while $2(2.4 \%)$ had a PB. Based on the postoperative mBP index, all $(100 \%)$ the patients had normal patellar height. None of the patients was found to have a patella alta postoperatively using any index.

\section{DISCUSSION}

We analyzed the effect of complete removal of IPFP during TKA on the patellar height, preoperatively, and at I y postoperatively. The preoperative average patellar height was higher than the value at I y postoperatively, using all measurement methods. Although this may reflect patellar tendon shortening or elevation of the joint line, this decrease was not significant using any method ( $p>0.05$ ). Our study indicates that complete removal of the IPFP during TKA had no significant effect on the height of the patella.

The removal or preservation of IPFP during TKA remains controversial $(3-5,8)$. Various studies have shown that complete removal of IPFP can cause patellar tendon shortening $(2,5,11)$. Tanaka et al. (II) observed a significant decrease in the length of the patellar tendon in 54 patients with rheumatoid arthritis who underwent IPFP removal than in 54 patients whose IPFP was preserved. A significant shortening of the patellar tendon in the IPFP removal group was shown by Lemon et al. (2). Chougule et al. (5) showed the shortening effects of IPFP removal during TKA. Compared to preoperative measurements, the length of the patellar tendon at I year was significantly shortened in I33 knees on which TKA with IPFP resection was performed (5).

In contrast, Macule et al. (9) stated that IPFP removal did not appear to change the patellar tendon length during the first 6 mon after TKA. Imren et al. (10) found similar results; the IPFP was completely removed in 228 patients who underwent TKA. They showed that IPFP removal during TKA did not alter the length of the patellar tendon at the 5-year follow-up (I0). In a recent study, patellar tendon length and functional outcome scores after TKA for osteoarthritis were compared for patients in whom IPFP was completely removed and those for whom it was preserved. No significant differences were observed in the IS, $\mathrm{mIS}, C D$, and $B P$ indices preoperatively, at $I y$, and at $5 y$ after the procedure between the resection group and preservation group. In our study, the length of the patellar tendon (evaluated using IS and $\mathrm{mIS}$ ratios) and height of the patellar (evaluated using CD and BP indices) were evaluated radiographically in patients who under- went complete IPFP removal during TKA. Complete removal of IPFP during TKA did not affect the length of the patellar tendon or the height of the patella at the l-year follow-up.

PB is described as a distally positioned patella relative to its normal position, measured using lateral radiography. It results from shortening of the patellar tendon or elevation of the joint line after TKA (6). PB is classified as true PB or false PB (6). True $\mathrm{PB}$ is caused by shortening of the patellar tendon, and it results in the pathological values of the indices (IS index $<0.8$ or mIS index<1.2 and $B P$ index $<0.54$ or $C D$ index $<0.6)(5-6,18)$. The pseudo-PB causes elevation of the joint line without shortening of the patellar tendon and causes changes in the $C D(<0.6)$ or BP $(<0.54)$ values. Moreover, the IS and $\mathrm{mIS}$ ratios remain normal (no patellar tendon shortening). IS and mIS ratios reflect the length of the patellar tendon, while the BP and CD indices show the distance between the distal pole of the patella and the tibial plateau $(5,6,18)$.

In our study, I (I.2\%) patient had true PB, while $4(4.8 \%)$ had pseudo-PB during the preoperative period. Moreover, 2 (2.4\%) of the patients had true PB, while another $2(2.4 \%)$ had pseudo-PB at the I-year follow-up. Pseudo-PB is a common complication after TKA and occurs when the patellar tendon is not shortened, but the level of the joint line increases. A large polyethylene insert used after tibial or femoral excessive resection to restore knee stability or excessive soft tissue release may also result in pseudo-PB (6). In a recent study, the incidence of pseudo-PB after TKA was 14.4\%; however, this was contradictory to our findings (6). However, Gatha et al. did not report a significant difference between the preoperative and postoperative patella height (19).

Chougule et al. (5) reported lengthening of the patellar tendon (according to IS ratio) in 133 knees of 16 (12.1\%) patients who underwent TKA with IPFP. They stated that this was owing to preoperative pain and disability caused by the degenerative process; in other words, the corrected patellar tendon shortened, as evidenced by improved postoperative functioning. In our study, patellar height increased in 27 patients (31.76\%) as per the IS ratio and in 29 (34.1I\%) patients as per the $\mathrm{mIS}$ ratio at the l-year follow-up. These increases reflect the lengthening of the patellar tendon. The patellar height increased in $32(37.6 \%)$ patients as per the $C D$ index and in $34(40 \%)$ patients as per the $\mathrm{BP}$ index at the l-year follow-up. These increases reflect a decline in the joint line and can be explained by shortening of the patellar tendon and a reduction in the patella height owing to the degenerative process in the preoperative knee joint. Thus, the patella height that had already decreased preoperatively increased after TKA. In fact, this increase indicated that the patella height returned to the normal value.

Our study has some strengths. The surgeries were performed by a single surgeon, while the same implant and technique were used for all the patients. Our study also has certain limitations. The study was designed retrospectively, the sample size was small, and the results were presented at the I-year follow-up. Further, we did not perform a comparison between early and late postoperative radiographs. In addition, no other group was included for the comparison, such as patients who underwent partial removal or preservation of the IPFP. 
In conclusion, we conclude that complete removal of the IPFP during TKA for primary osteoarthritis did not affect the patellar height at the l-year follow-up. Larger case series and/or randomized controlled studies are needed to confirm our results.

Ethics Committee Approval: Ethics committee approval was received for this study from the ethics committee of Aksaray University (2020/06-12).

\section{Informed Consent: N/A}

Peer-review: Externally peer-reviewed.

Author contributions: Concept - S.G., S.C., R.Ö.; Design - S.G., S.C.; Supervision - S.G., S.C., R.Ö.; Resource - S.G., S.C.; Materials - S.G., S.C.; Data Collection and/or Processing - S.G., S.C.; Analysis and/or Interpretation - S.G., S.C.; Literature Search - S.G., S.C., R.Ö.; Writing - S.G., S.C., R.Ö.; Critical Reviews - S.C., R.Ö.

Conflict of Interest: Authors have no conflicts of interest to declare.

Financial Disclosure: The authors declared that this study has received no financial support.

\section{REFERENCES}

I. Gallagher J, Tierney P, Murray P, O'Brien M. The infrapatellar fat pad: anatomy and clinical correlations. Knee Surg Sports Traumatol Arthrosc 2005; 13(4): 268-72. [Crossref]

2. Lemon M, Packham I, Narang K, Craig DM. Patellar tendon length after knee arthroplasty with and without preservation of the infrapatellar fat pad. J Arthroplasty 2007; 22(4): 574-80. [Crossref]

3. Gwyn R, Kotwal RS, Holt MD, Davies AP. Complete excision of the infrapatellar fat pad is associated with patellar tendon shortening after primary total knee arthroplasty. Eur J Orthop Surg Traumatol 2016; 26(5): 545-9. [Crossref]

4. Ye C, Zhang W, Wu W, Xu M, Nonso NS, He R. Influence of the infrapatellar fat pad resection during total knee arthroplasty: a systematic review and meta-analysis. PLoS One 2016; II(I0): e0163515. [Crossref]

5. Chougule SS, Stefanakis G, Stefan SC, Rudra S, Tselentakis G. Effects of fat pad excision on length of the patellar tendon after total knee replacement. J Orthop 2015; 12(4): 197-204. [Crossref]
6. Aguirre-Pastor A, Ortolá DJ, Lizaur-Utrilla A, Rosa MA, Lopez-Prats FA. Is pseudo-patella baja really a serious complication of total knee arthroplasty? J Arthroplasty 2020; 35(2): 557-62. [Crossref]

7. Rogers BA, Thornton-Bott P, Cannon SR, Briggs TW. Interobserver variation in the measurement of patellar height after total knee arthroplasty. J Bone Joint Surg Br 2006; 88(4): 484-8. [Crossref]

8. Yao B, Samuel LT, Acuña AJ, Faour M, Roth $A$, Kamath $A F$, et al. Infrapatellar fat pad resection or preservation during total knee arthroplasty: a systematic review. J Knee Surg 2019 doi: 10.1055/s0039-1696692. Online ahead of print. [Crossref]

9. Maculé F, Sastre S, Lasurt S, Sala P, Segur JM, Mallofré C. Hoffa's fat pad resection in total knee arthroplasty. Acta Orthop Belg 2005; 7|(6): 7|4-17.

10. İmren Y, Dedeoğlu SS, Çakar M, Çabuk H, Bayraktar TO, Gürbüz H. Infrapatellar fat pad excision during total knee arthroplasty did not alter the patellar tendon length: a 5-year follow-up study. J Knee Surg 2017; 30(5): 479-83. [Crossref]

II. Tanaka N, Sakahashi H, Sato E, Hirose K, Isima T. Influence of the infrapatellar fat pad resection in a synovectomy during total knee arthroplasty in patients with rheumatoid arthritis. J Arthroplasty 2003; 18(7): 897-902. [Crossref]

12. Insall J, Salvati E. Patella position in the normal knee joint. Radiology 1971; 10I(I): 10I-4. [Crossref]

13. Grelsamer RP, Meadows S. The modified Insall-Salvati ratio for assessment of patellar height. Clin Orthop Relat Res 1992; 282: 170-6. [Crossref]

14. Blackburne JS, Peel TE. A new method of measuring patellar height. J Bone Joint Surg Br. 1977; 59(2): 24I-2. [Crossref]

15. Caton J, Deschamps G, Chambat P, Lerat JL, Dejour H. Apropos of 128 cases. Patella infera. Rev Chir Orthop Reparatrice Appar Mo† 1982; 68(5): 317-25.

16. Classen T, Wegner A, con Knoch M. Modification of the Blackburne-Peel ratio for improved application in total knee arthroplasty. Orthopade 2009; 38(12): 1229-34. [Crossref]

17. Shrout PE, Fleiss JL. Intraclass correlations: uses in assessing rater reliability. Psychol Bull 1979; 86(2): 420-8. [Crossref]

18. $X \cup B, X \cup W X, L \cup D$, Sheng HF, Xu XW, Ding WG. Application of different patella height indices in patients undergoing total knee arthroplasty. J Orthop Surg Res 2017; 12(I): 191. [Crossref]

19. Gatha NM, Clarke HD, Fuchs R, Scuderi GR, Insall JN. Factors affecting postoperative range of motion after total knee arthroplasty. J Knee Surg 2004; 17(4): 196-202. [Crossref] 